\title{
AISLAMIENTO SOCIAL POR COVID-19 EN LA AFECTACIÓN DE LA SALUD MENTAL: CASO UNIVERSIDAD DE LA GUAJIRA
}

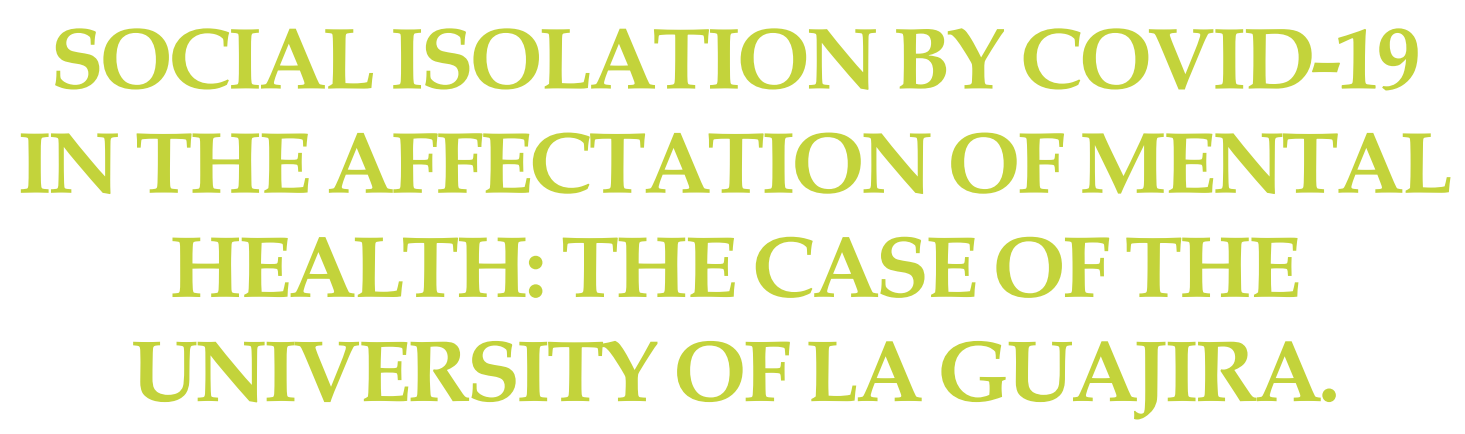

Clara Judith Brito Carrillo ${ }^{1}$

Elvis Eliana Pinto Aragón²

Delvis Muñoz Rojas ${ }^{3}$

Universidad de La Guajira

$1 \quad$ Mcs. Desarrollo y Gestión de empresas sociales Gerencia Social. Trabajadora Social, Docente Universidad de la guajira. Investigador asociado, líder Grupo de Investigación: Encuentro con la investigación Categorizado en A1. la Guajira. Investigador asociado. Autor y coautor de artículos de revistas científicas e indexadas, capítulo de libros, libros a nivel nacional e internacional. 2016. escenarios@ trabajosocial.unlp.edu.arl.Email.clarabrito@uniguajira.edu. co. https://orcid.org/0000-0001-8788-7326

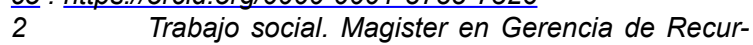
sos Humanos. Docente e investigadora de la Universidad de La Guajira Colombia. Autor y coautor de artículos de revistas científicas e indexadas, capítulo de libros, libros a nivel nacional e internacional. Grupo de Investigación: Encuentro con la investigación Categorizado en A1 Email:epinto@ uniguajira.edu.co.https://orcid.org/0000-0002-9866-9751

3 Dhp en Ciencia Mención Gerencia, Mgs en Sistema de Gestión, Especialista en Salud Ocupacional y Finanzas, Psicóloga, y Administradora de empresa, investigador senior. Grupo de Investigación: Encuentro con la investigación Categorizado en A1. Autor y coautor de artículos de revistas científicas e indexadas, capítulo de libros, libros a nivel nacional e internacional. Email: dmunozr@uniguajira. edu.co, https://orcid.org/0000-0001-9445-9792

\section{RESUMEN}

El artículo tuvo como objetivo analizar los estragos del aislamiento social por COVID-19 en la salud mental de los estudiantes de la Universidad de La Guajira, Colombia. Metodológicamente, esta investigación es de corte cualitativo, descriptivo y fenomenológico; la población se caracterizó por una muestra de veinte estudiantes, lo cual permitió realizar un análisis profundo a través de las experiencias e historias de vida de diversos estudiantes. A partir de estas es posible identificar la aparición de diversos síntomas relacionados con daños en la salud mental, considerando sus estados psicológicos y sociales. A manera de conclusión se denota 
una multiplicidad de distintas consecuencias en el ámbito educativo, dado que los estudiantes manifestaron una desmotivación para seguir estudiando, debido a la falta de herramientas y habilidades tecnológicas tanto por parte de ellos como de los docentes. La calidad educativa, así como el rendimiento escolar, disminuyó en las modalidades implementadas por las instituciones, dejando a los alumnos con una carga de trabajo mayor al conocimiento adquirido durante la impartición de materias; surgió en parte de la comunidad estudiantil la idea de abandonar los estudios a causa de las limitantes ya mencionadas.

\section{PALABRAS CLAVE}

Afectación, aislamiento social, covid-19, salud mental

\section{ABSTRACT}

The objective of the article was to analyze the ravages of social isolation by covid-19 on the mental health of students at the university of la guajira, colombia, methodologically, this research is qualitative, descriptive and phenomenological, the population was characterized by a sample of twenty students, which allowed a deep analysis through the experiences and life stories of various students, from these it is possible to identify the appearance of various symptoms related to damage to mental health, considering their psychological and social states. By way of conclusion, a multiplicity of different consequences in the educational field is denoted, since the students manifested a lack of motivation to continue studying, this due to the lack of tools and technological skills on the part of both them and the teachers, the educational quality as well as the school performance decreased in the modalities implemented by the institutions leaving the students with a workload greater than the knowledge acquired during the teaching of subjects, the idea of abandoning their studies arose in part of the student community because of the aforementioned limitations.

\section{KEY WORDS}

Affectation, social isolation, covid-19, mental health

\section{INTRODUCCIÓN}

Desde diferentes perspectivas, la aparición de un nuevo virus identificado como SARS-CoV2 causó un gran impacto en la humanidad ya que rompió con gran parte de los esquemas cotidianos en la vida de las personas, generando un colapso en diferentes áreas de la sociedad, entre ellas la educativa. Cuando el número de contagios aumentó de manera considerable se tomaron medidas para disminuirlo, para ello se recurrió al confinamiento. La cuarentena, como medida de prevención, aisló a las personas dentro de sus hogares, imponiendo una nueva realidad a través de la distancia social, sin embargo, esto implicó múltiples factores de riesgo para la salud mental de la sociedad.

Los resultados de la siguiente investigación nos indican que a causa del aislamiento social por COVID-19 se generaron diversos estragos en la salud mental de los universitarios pertenecientes a la Universidad de La Guajira ubicada en Colombia. A través de cuestionarios en línea, así como entrevistas y conversatorios con universitarios se encontró que al enfrentarse a esta situación surgieron daños en su salud mental entre los cuales destacan los siguientes: ansiedad, depresión, crisis nerviosas, insomnio y estrés; además de que sus relaciones intrafamiliares e interpersonales en la mayoría de los casos resultaron afectadas de manera negativa. En un recorrido por sus experiencias se logró identificar distintos factores de riesgo en el área económica, psicológica, social, educativa y biológica.

En este orden de ideas, La Organización Mundial de la Salud (OMS, 2020) señala que "Se puede 
determinar que la salud mental es el estado dinámico expresado en la vida cotidiana por el comportamiento y la interacción que analiza la salud psíquica, como un estado que involucra a varios componentes positivos o negativos, dependiendo su ánimo y actitud". Ante esto es importante reconocer que la llegada de un fenómeno con imprevisto que rompe con los esquemas con los cuales se rige la vida de diversas sociedades genera un estado de crisis e inestabilidad permanente ante la incertidumbre del futuro y del presente mismo.

No obstante, el confinamiento como medida preventiva tiene a su vez consecuencias negativas para la salud mental de las personas, la imposición de una nueva realidad que implica una adaptación inmediata en diversos ámbitos de la vida diaria, la modificación de los modos de relacionarse con otras personas así como factores de riesgo que se incrementan principalmente económicos y de salud, generan en los individuos emociones, sentimientos y pensamientos con cargas negativas, que afectan de manera significativa su existencia.

La adaptación de los estudiantes pertenecientes al nivel superior a los nuevos modelos de enseñanza-aprendizaje implicó un desafío ya que fue un proceso espontáneo, el cual implicaba la necesidad de contar con los medios tecnológicos necesarios para mantenerse en contacto con sus docentes, un manejo de las Tecnologías de la Información y la Comunicación (TICs) para sostener sus estudios, al igual que contar con los recursos económicos suficientes para acceder a estas. Las preocupaciones por parte de los estudiantes para resolver dichos asuntos, así como los factores de riesgo a los que se enfrentaban en diversas esferas de su vida significó una desestabilización cognitiva y anímica que más tarde se manifestó con diversos síntomas.

Una vez aplicada la metodología correspondiente se encontró que los universitarios y universitarias en su vida académica durante el aislamiento padecieron estrés, ansiedad, insomnio, incertidumbre, etc., Así mismo, la idea de desertar de los estudios a causa de la deficiencia en calidad de enseñanza en sus nuevas modalidades fue considerada por algunos estudiantes. Esto nos demuestra la importancia de la acción de intervención y prevención multidisciplinaria por parte de las instituciones académicas para atender las diversas situaciones de riesgo a la que se enfrentan sus estudiantes.

La enfermedad COVID-19 es causada por el SARS-CoV-2. Virus de este tipo, El virus de este tipo cuando se ha transferido de animales a los humanos liderados en los últimos años, dieron origen a las epidemias del Síndrome Respiratorio Agudo Severo y el Síndrome Respiratorio del Medio Oriente (Shereen et al., 2020). El primer diagnóstico De este nuevo coronavirus se llevó a cabo a fines de diciembre de 2019 en Wuhan, China. El virus se extendió rápidamente y se identificó el primer caso fuera de China, en enero de 2020 (Wu \& McGoogan, 2020).

\section{DESARROLLO}

Según la Organización de las Naciones Unidas para la Educación, la Ciencia y la Cultura (UNESCO, 2020), expresa que:

Según la Unión Internacional de Telecomunicaciones de las Naciones Unidas, antes del brote de COVID-19 sólo el $47 \%$ de la población delospaísesen desarrollo utilizaba Internet, en comparación con el $86 \%$ de la población en los países desarrollados. Como señala la Cátedra de Cultura de Paz y Educación de la Universidad Técnica Particular de Loja (Ecuador) "al menos el $60 \%$ de la 
población estudiantil se ha visto afectada por la pandemia, ya que muchos no tienen los medios ni los instrumentos para acceder a la enseñanza en línea. Dado que COVID-19 está teniendo graves repercusiones sobre los estudiantes, se necesitan respuestas oportunas

En vista de lo anterior, es importante denotar que el COVID-19, rompió con los esquemas tradicionales a nivel mundial, generándose una crisis global que ha inestabilizado los sectores salud, educativos, políticos, sociales y familiares, en consideración se puntualiza que a fínales del año 2019 surgió un brote de Coronavirus en China que más tarde se confirmó su origen por la transmisión del virus 2019-nCoV denominado así por la Organización Mundial de la Salud (OMS,2019).

En este orden de ideas, después de algunos meses se propagó por varios países de Asia, Europa y América, generando así la Pandemia de COVID-19. Las medidas preventivas que se tomaron para disminuir la tasa de contagio implicaron un aislamiento social casi total, su alcance fue tal que se rompieron los modos de acción en diversos sectores de la población, desde el laboral hasta el académico lo cual ha significado un gran reto tanto para los individuos como para las instituciones, quienes han buscado adaptarse a la nueva normalidad.

Por su parte, la educación a distancia se implementó a partir del establecimiento de cuarentena en los países afectados por la pandemia, entre ellos se encuentran Colombia, esta modalidad en la educación implica la impartición de clases en formato en línea, los estudiantes viven el proceso de enseñanzaaprendizaje a través de las pantallas de los aparatos tecnológicos que tienen a su alcance en sus hogares, esta adaptación de sus tareas cotidianas supone procesos mentales que pueden influir en los estados anímicos de los universitarios así como favorecer el deterioro de la salud mental ante el miedo y la incertidumbre que trae consigo la pandemia.

Dentro de este contexto, el periódico Heraldo (2020), plantea que:

Uno de los impactos a corto plazo que se registra en el caso de los alumnos es la reorganización de su vida cotidiana para ajustarse a una situación de confinamiento. "Inevitablemente, la pérdida de contacto social y de las rutinas de socialización que forman parte de la experiencia cotidiana de un estudiante de educación superior tendrán un costo", señala el informe. La consecuencia central del aislamiento es la "sustitución de las clases presenciales" y la necesidad de los estudiantes de adaptarse a nuevas formas de enseñanza $y$ aprendizaje bajo una modalidad virtual. En ese sentido, una gran parte de los alumnos no han recibido dicho cambio de manera muy positiva.

En otras palabras, en parte porque "el contenido que se ofrece nunca fue diseñado en el marco de un curso de educación superior a distancia, sino que intenta paliar la ausencia de clases presenciales con clases virtuales sin mayor preparación previa". Además "la educación a distancia requiere de mayor disciplina $y$ compromiso por parte del estudiante, lo que quizás explique que ésta tenga más éxito entre alumnos de mayor edad, esto es, los de posgrado". Los estudiantes de zonas vulnerables son los más perjudicados ya que el cambio de modalidad les exige conectividad a internet y en muchos casos no tienen acceso a ese servicio. Por eso, una de las preguntas centrales que se 
formula el informe es "si en el supuesto de la continuidad de las actividades de enseñanza, los estudiantes conseguirán lograr los objetivos de aprendizaje diseñados para el curso.

En vista de lo anterior, el aislamiento social producido por el COVID-19, posee su impacto negativo en la sociedad, lo cual ha generado estragos en la salud mental de los universitarios. ¿De qué manera se evidencian los estragos del aislamiento social por COVID-19 en la salud mental de los universitarios? En este sentido, desde las perspectivas del perfil profesional de Trabajo Social y Psicología, han enfrentado a nuevos retos desde su conciencia transformadora en lo económico, político y social partiendo desde las bases teóricas la cual fundamenta su quehacer con un enfoque humanista dada la ubicación geoestratégica del departamento de La Guajira, pluriétnico y multicultural.

Cuadro No 1.- Unidades de análisis que responden a la investigación

\begin{tabular}{|c|c|}
\hline \multicolumn{2}{|c|}{$\begin{array}{l}\text { ASPECTOS GENERALES DE LOS ESTUDIANTES DE LAS UNIVERSIDAD DE LA GUAJIRA } \\
\text { COLOMBIA }\end{array}$} \\
\hline $\begin{array}{l}\text { Dificultades al acceso a las oportunidades } \\
\text { de aprendizaje dadas las condiciones } \\
\text { socioeconómicas de los estudiantes }\end{array}$ & $\begin{array}{l}\text { Ansiedad -depresión. No aceptación. } \\
\text { Garantizar una enseñanza efectiva y de } \\
\text { calidad }\end{array}$ \\
\hline $\begin{array}{l}\text { Las Universidades se enfrentan a serios } \\
\text { desafíos para alcanzar los indicadores } \\
\text { académicos con relación a las poblaciones } \\
\text { más vulnerables. }\end{array}$ & $\begin{array}{l}\text { Implementación de estrategias } \\
\text { Flexibilidad academia } \\
\text { Promover discusiones informadas sobre temas } \\
\text { educativos }\end{array}$ \\
\hline $\begin{array}{l}\text { Pérdida temporal del espacio de protección } \\
\text { que ofrecen los entornos Universitarios. }\end{array}$ & $\begin{array}{l}\text { Inestabilidades. debilitamiento en las } \\
\text { relaciones personales e interpersonales. }\end{array}$ \\
\hline $\begin{array}{l}\text { Factores de riesgo psicosociales del } \\
\text { aislamiento social por COVID-19 }\end{array}$ & $\begin{array}{l}\text { Afectación a la salud mental de los estudiantes } \\
\text { Crisis en el aprendizaje }\end{array}$ \\
\hline Covid-19 una Crisis global & $\begin{array}{l}\text { Inestabilidad económica-política-social- } \\
\text { educativa-salud }\end{array}$ \\
\hline Total & Veinte (20) Estudiantes \\
\hline
\end{tabular}

Fuente: investigadores, 2020

Peiró (2021, 5 de enero) señala que la Psicología de la Salud:

Está interesado en la conducción, la actitud y los procesos que se producen en un individuo para influir en su salud. $Y$ con respecto a esto con cualquier patología existente, que, por ejemplo, desarrollada o desarrollada como ansiedad o depresión.

Por su parte, la atención desde una mirada multidisciplinar permite entender lo que sucede en las personas de una mejor manera, ya que brinda la información necesaria en la cual se puede intervenir para mantener una calidad de vida en la cual su salud mental es de gran importancia. Cullari (2001) explica que:

El campo de la psicología
clínica integra ciencia, teoría y
práctica para entender, predecir
y aliviar el desequilibrio, la
invalidez y la incomodidad;
también promueve la adaptación
humana, el ajuste y el desarrollo
personal. La psicología clínica
está enfocada en los aspectos
intelectuales, emocionales,


biológicos, psicológicos, sociales y del comportamiento humano que funcionan a través de la existencia en las diferentes culturas, $y$ en todos los niveles socioeconómicos.

(p.2)

Salud: La Real Academia Española (2016) define el concepto de salud como un "estado, en el que el ser orgánico generalmente ejerce todas las funciones" y "un conjunto de condiciones físicas, en las que un organismo está en un momento determinado". Por lo general, la gente considera que si no se enferma físicamente son "saludables", sin embargo, se han descubierto en nuestros tiempos en que nuestro bienestar depende de la cantidad de autocuidado que tenemos en nuestros diferentes roles y contextos.

Estrés: El estrés es el conjunto de reacciones presentadas por el cuerpo humano cuando se expone a situaciones estresantes y que se pueden manifestar como sentimientos de abrumador, preocupación y agotamiento. Por lo tanto, una persona puede sentirse cuando se expone a cualquier agente estresor (Malave, 2020)

Entre tanto, OMS (2020) señala que el estrés en tiempo de pandemia presenta muchas dificultades personales entre las que se puede mencionar conflictos con familiares, soledad, falta de ingresos, preocupación por el futuro), Problemas en el trabajo (por ejemplo, conflictos con colegas, necesidades de alto nivel $O$ el trabajo no es seguro) o existen grandes amenazas en la comunidad (por ejemplo, Violencia, enfermedad, falta de oportunidades económicas).

Miedo: Así mismo, Valero, Vélez, Durán, y Portillo (2020). Dicen que el miedo es una emoción adaptativa que sirve a la energía para movilizarla y enfrentar una amenaza con valor. Sin embargo, si el miedo no está calibrado para la amenaza real, puede ser desadaptivo. Si el miedo es muy excesivo, esto puede estar a nivel individual (problemas de salud mental, fobias y ansiedad) a nivel social (compras de pánico, xenofobia, entre otros.).

Depresión: La depresión, definida como un trastorno mental y emocional $\mathrm{Li}$ et al (2015). Crea una combinación de factores biológicos, psicológicos y sociales. (Ellis et al, 2017); y constituye un factor de riesgo en ancianos (65 años y más), en donde la soledad se asume como un factor psicosocial de riesgo permanente en ellos.

Trastornos de la afectividad El trastorno afectivo es un trastorno mental, recurrente e incapacitante se encuentra en las alteraciones del estado de ánimo, se caracteriza por episodios de cambios en las emociones, el conocimiento y los comportamientos, para la mayoría de las personas, estos cambios les permiten ejercer un cierto grado de control sobre sus propios sentimientos. Sin embargo, otros no pueden hacerlo, interfiere significativamente en todas las áreas de sus vidas (Martínez y Paladinez, 2016).

Preocupación: Las preocupaciones se dan cuando creemos que algo falta en nuestras vidas y se sale de nuestro control, o no está como nos gustaría. Así que un pensamiento en nuestra cabeza aparece recurrente y circular sin que sea esta la solución. De hecho, lejos de ayudarnos a comenzar a bloquearnos y hacernos ansiosos. Si trabajamos para detener los pensamientos y las preocupaciones, podemos comenzar a generar alternativas y reducir los síntomas de ansiedad. (Rull, 2019).

\section{RESULTADO DE INVESTIGACIÓN}

\section{TIPO DE INVESTIGACIÓN:}

El tipo de investigación que se utilizó en esta investigación fue el descriptivo e interpretativo, 
El primero de ellos se enfoca en recabar las narraciones o descripciones de los sujetos que forman parte del estudio, respetando en todo momento sus propias percepciones acerca del fenómeno estudiado. En cambio, el estudio interpretativo o hermenéutica -como su nombre lo indica- elabora interpretaciones de la experiencia estudiada con base en lo que los participantes expresan (Hernández, et al., 2014).

\subsection{ENFOQUE METODOLÓGICO}

Según Ramos (2017), el enfoque cualitativo busca la comprensión de los fenómenos en su ambiente usual, desarrollando la información basada en la descripción de situaciones, lugares, periódicos, textos, individuos, etc.,

El enfoque a utilizar en este estudio es el cualitativo. En ese sentido, "los métodos cualitativos son utilizados para estudiar cualquier grupo humano, cuyas relaciones están reguladas por la costumbre o por ciertos derechos y obligaciones reciprocas como familia, instituciones educativas, etc.". (Hernández y Coello, 2011, p. 86).

\section{DISEÑO DE INVESTIGACIÓN:}

Atendiendo las recomendaciones de los autores citados, dentro de los diferentes métodos que están dentro de la metodología cualitativa destaca el método fenomenológico el objetivo que persigue es la comprensión de la experiencia de vivir en su complejidad; esta comprensión a su vez busca la conciencia y el significado del fenómeno. Para utilizar este método de investigación, es necesario comprender los conceptos y principios de la fenomenología, así como el método para ingresar al campo de la investigación y el mecanismo de búsqueda de significado. Comprender la experiencia a través de relatos, historias y anécdotas es esencial porque nos permite comprender la naturaleza de la dinámica contextual e incluso cambiarla (Fuster, 2019. pp.221-229)

El diseño utilizado en este trabajo fue el fenomenológico porque propende comprender los fenómenos como son, como se viven permitiéndoles que se desarrollen por sí mismos, sin imponer un marco desde afuera, sino que por el contrario respetando su naturaleza (Martínez 2010). La fenomenología "considera que los seres humanos están vinculados con el mundo y pone el énfasis en su experiencia vivida, la cual aparece en el contexto de las relaciones con objetos, personas, sucesos y situaciones" (Álvarez, 2003, p. 86). Para Husserl (como se citó en Martínez, 2010), La elección de este enfoque para este proceso investigativo permitirá conocer las actitudes y comportamientos producidos en la salud mental de los universitarios. Estudio de caso Universidad de La Guajira Colombia y Benemérita Universidad Autónoma de Puebla México

\section{POBLACIÓN Y MUESTRA}

Según, Monje (2011) "la muestra se define como un conjunto de objetos y sujetos procedentes de una población, es decir, un subgrupo de la población cuando esta es definida como un conjunto de elementos que cumplen con unas determinadas especificaciones". Por otra parte, las muestras deben ser representativas, es decir, deben reflejar o representar las características de la población de donde provienen lo que garantiza que los resultados son aplicables o inferirle a dicha población. (p.123)

Cabe destacar, que las muestras deben ser cualitativamente buenas, es decir, que sean homogéneas lo que indica que deben ser de una misma población; adecuadas porque debe incluir todas las variaciones que en las características de la población puedan tener; y finalmente es necesario que sean no viciadas en cuanto a que no deben presentar sesgo en su selección lo 
que desencadenaría en invalidar los resultados por más precisos que sean los instrumentos.

Según, Berenguera, Fernández, Pons, Pujol, Rodríguez y Saura (2014) la muestra en investigación cualitativa debe dirigirse a seleccionar aquellos participantes que pueden dar información significativa, rica, completa y profunda sobre la compresión del fenómeno de estudio

\section{TECNICA DE RECOLECCION DE DATOS}

Las técnicas más utilizadas en fenomenología son: a) Observación y observación participante; b) conversaciones informales y c) entrevistas a profundidad, en esta investigación se utilizó la técnica de entrevista en profundidad.

\section{ENTREVISTA EN PROFUNDIDAD: La} apropiación de esta técnica es conocer a grandes rasgos la incidencia misma de propio consumidor y personas afectadas por el consumo de sustancias psicoactiva, su aplicabilidad contara con momentos de verdad y de gran significados en el cual cada persona transmitirá oralmente al ser entrevistado su visión personal de la situación En el caso de estudios cualitativos, específicamente en las entrevistas en profundidad, se identifican categorías o grupos de conceptos relevantes para la investigación, con la finalidad de comprender, interpretar, reconstruir y reflexionar acerca de las experiencias e historias de los informantes (Arias. 2012. p. 137)

* Historias de Vida. La técnica de historia de vida: según, Chárriez (2012), "la historia de la vida es una técnica cuyo objetivo es "comprender el comportamiento humano de su propio marco de referencia"(P.51), además, indica que los recuerdos de la gente, las historias de vida suelen construirse también con la ayuda de cartas, informes de la época o descripciones de periódicos para ampliar la información que da el individuo y tener un parámetro de comparación (Giddens, 1998).

* Estudios de casos:

Un estudio de caso trata con éxito una situación técnicamente inconfundible, en la que muchas más variables de interés son datos observacionales, $y$, como resultado, basados en múltiples fuentes de prueba, con datos que deben converger en un estilo de triangulación; Además, se beneficia de los problemas teóricos del desarrollo previo que dirigen la recopilación y el análisis de datos (Monge, 2010).

Según, Yin (1994) señala que una de las justificaciones válidas para estudios de caso sobre experiencias singulares proviene de su carácter único o raro y de su capacidad reveladora en un área teórica específica, actuar efectivamente en el logro de los mismos. El análisis se centra por lo general en la interacción entre actores que persiguen intereses identificados con cierto grado de objetividad por el investigador.

En la presente investigación, en primer término, se explica este parámetro dentro del proceso que se sigue en la investigación cualitativa. Para ello Gento y Huber (2012) mencionan que la fiabilidad no procede directamente de una medida, sino que viene dada por la constancia en la aparición de los tipos o categorías de hechos o fenómenos observados por lo que será importante una debida acotación de estos hechos o fenómenos. Es estos conceptos mencionados en la presente investigación, la fiabilidad o confiabilidad de la misma viene representada en la parte cualitativa en los siguientes elementos que se presentan en el cuadro 
Tabla 2 Elementos de fiabilidad cualitativa

\begin{tabular}{|l|l|}
\hline $\begin{array}{l}\text { ELEMENTO DE FIABILIDAD EN } \\
\text { LO CUALITATIVO }\end{array}$ & DESCRIPCIÓN \\
\hline Validez descriptiva & $\begin{array}{l}\text { Transcripción de las entrevistas, anotaciones, documentos, } \\
\text { grabaciones realizadas a los estudiantes universitarios }\end{array}$ \\
\hline Validez interpretativa & $\begin{array}{l}\text { Concertar una reunión virtual con los estudiantes para que } \\
\text { accedan a la aplicabilidad del estudio de caso para tener } \\
\text { claridad sobre los acontecimientos de la investigación }\end{array}$ \\
\hline Validez teórica & $\begin{array}{l}\text { Está relacionada con todas las construcciones de las bases } \\
\text { teóricas referenciales desarrolladas en la investigación }\end{array}$ \\
\hline Transferibilidad & $\begin{array}{l}\text { Los resultados de la presente investigación pueden } \\
\text { transferirse a otros contextos donde se estudie la misma } \\
\text { categoría de investigación. }\end{array}$ \\
\hline Replicabilidad & $\begin{array}{l}\text { Se puede repetir la investigación y obtener los mismos } \\
\text { resultados, cuando se mantienen las mismas condiciones, } \\
\text { lo que permite de esta manera redundar en la solidez del } \\
\text { estudio }\end{array}$ \\
\hline
\end{tabular}

Elaboración investigadores Brito Carrillo, Pinto Aragón y Muñoz Rojas, 2021

\section{DISCUSIÓN DE RESULTADOS \\ CASO 1 -}

\begin{tabular}{|l|l|}
\hline Datos & Experiencia a través del aislamiento social por COVID-19 \\
\hline Género: & ¿Cómo ha influido el aislamiento social en tu vida universitaria? Difícil por los \\
Edad: 21 Años & medios de comunicación tecnológicos. \\
Estado civil: & ¿Consideras que el confinamiento por la pandemia de COVID-19 ha influido en \\
madre soltera & tus relaciones interpersonales? (Amigos/ conocidos/ compañeros de escuela/ \\
1 hijo & compañeros de trabajo) ¿Cómo? Distanciamiento físico solo eso. \\
Programa de & ¿Qué factores de riesgo económicos consideras que has enfrentado durante la \\
Trabajo social & contingencia? Económicos, y comerciales \\
Labora: No & ¿Cómo te has sentido respecto a tu vida universitaria durante la contingencia? \\
& $\begin{array}{l}\text { (Emociones/Sentimientos/Pensamientos) Me afectado la salud. Con crisis de } \\
\text { pánico } \\
\text { ¿Has pensado en desertar de la universidad a causa de la contingencia? ¿Por } \\
\text { qué? No } \\
\text { ¿Cómo definirías tu estado emocional frente al aislamiento social? Perjuicios de } \\
\\
\text { salud mental } \\
\text { ¿Tu universidad ha implementado programas de intervención ante crisis } \\
\text { psicológicas respecto al aislamiento social? ¿Cuáles? Si. Proyectos de } \\
\text { investigación } \\
\text { ¿Te encuentras satisfecho respecto a la nueva modalidad de enseñanza- } \\
\text { aprendizaje implementada por tu universidad? ¿Por qué? Si. Contribuye a seguir } \\
\text { adelante el proceso de formación }\end{array}$ \\
\hline
\end{tabular}

Elaboración investigadores Brito Carrillo, Pinto Aragón \& Muñoz Roja, 2021 


\section{ANÁLISIS CASO 1.-}

Manifiesta haber sufrido crisis de ansiedad y estrés durante el aislamiento social, originados a partir de problemas de salud y emocionales. Durante estas crisis menciona sufrir fiebre y sentir presión. Tomar medicamentos psiquiátricos para controlar sus crisis de ansiedad y para conciliar el sueño. En el ámbito familiar se presenta nerviosa, ya que tiene problemas familiares.

En el ámbito económico menciona que se ha visto afectada a causa del confinamiento, ya que no tiene trabajo lo cual repercute directamente en el abastecimiento de productos del hogar como alimento e insumos para sus hijos. En cuanto a los miedos que ha manifestado a causa de la Pandemia se encuentran el temor a morir, a enfermarse ya que sufre de una enfermedad crónica que la vuelve vulnerable ante el COVID-19, esto implica la preocupación por proteger a sus hijos.

Afectada, apoyo moral en el ámbito educativo, Pedagogía infantil. Dificultades, niños pequeños, apartarse de la universidad, Pandemia, trastornos, adaptación, no teníamos medios para comunicarnos. Crisis. Dificultades económicas.

Elaboración investigadores Brito Carrillo, Pinto Aragón \& Muñoz Roja, 2021

\begin{tabular}{|l} 
ANÁLISIS CASO 2.- \\
\hline Durante el conversatorio menciona que durante el confinamiento el contacto con las personas \\
que la rodean se rompió, sobre todo con los docentes de su universidad afirmando que la \\
relación estudiante docente es fundamental. Menciona que el lenguaje corporal, es fundamental \\
en su profesión (Trabajo Social). En el ámbito educativo a través del semestre virtual, no hay \\
conocimiento de docentes para enseñar a través de las tecnologías empleadas.
\end{tabular}

En cuanto a su estado anímico menciona que ha sido una "curva de emociones", al inicio, permaneció en la ciudad en la que residía ya que se encontraba trabajando, sin embargo, cuando decidió regresar a su lugar de origen en donde se encontraba su familia no logró realizarlo a causa de que habían cerrado el camino así que se quedó atrapada, esto le generó ansiedad, estrés e incertidumbre.

Cuando logró regresar a casa su estado de ánimo mejoró, pero con el paso del tiempo la convivencia familiar se tornó difícil ya que se presentaron situaciones problemáticas con los miembros de la familia. Además de esto la preocupación en su hogar aumentó a causa de que su padre sufre una enfermedad que lo posiciona entre las personas vulnerables en cuestión a la pandemia por COVID-19.

En la cuestión académica su rendimiento escolar se vio dificultado a causa de impedimentos para realizar sus actividades ya que en su hogar solo contaban con una computadora, y esta era utilizada por ella y sus dos hermanos lo cual no les permitía el cumplimiento de las metas

Su situación económica se volvió complicada ya que le suspendieron el apoyo económico con el que contaba por parte del estado además de que su papá perdió su trabajo, así que restringieron sus gastos para lograr adaptarse. Afirma que es fundamental el acompañamiento psicológico y el acompañamiento familiar, esto con el fin de brindar herramientas para sobrellevar las situaciones que se presentan. 


\section{CASO 3}

\begin{tabular}{|c|c|}
\hline Datos & Experiencia a través del aislamiento social por COVID-19 \\
\hline $\begin{array}{l}\text { Género: Masculino } \\
\text { Edad: } 18 \text { Años } \\
\text { Estado civil: Soltero } \\
\text { Programa Derecho } \\
\text { Convive con sus } \\
\text { padres Biológicos }\end{array}$ & 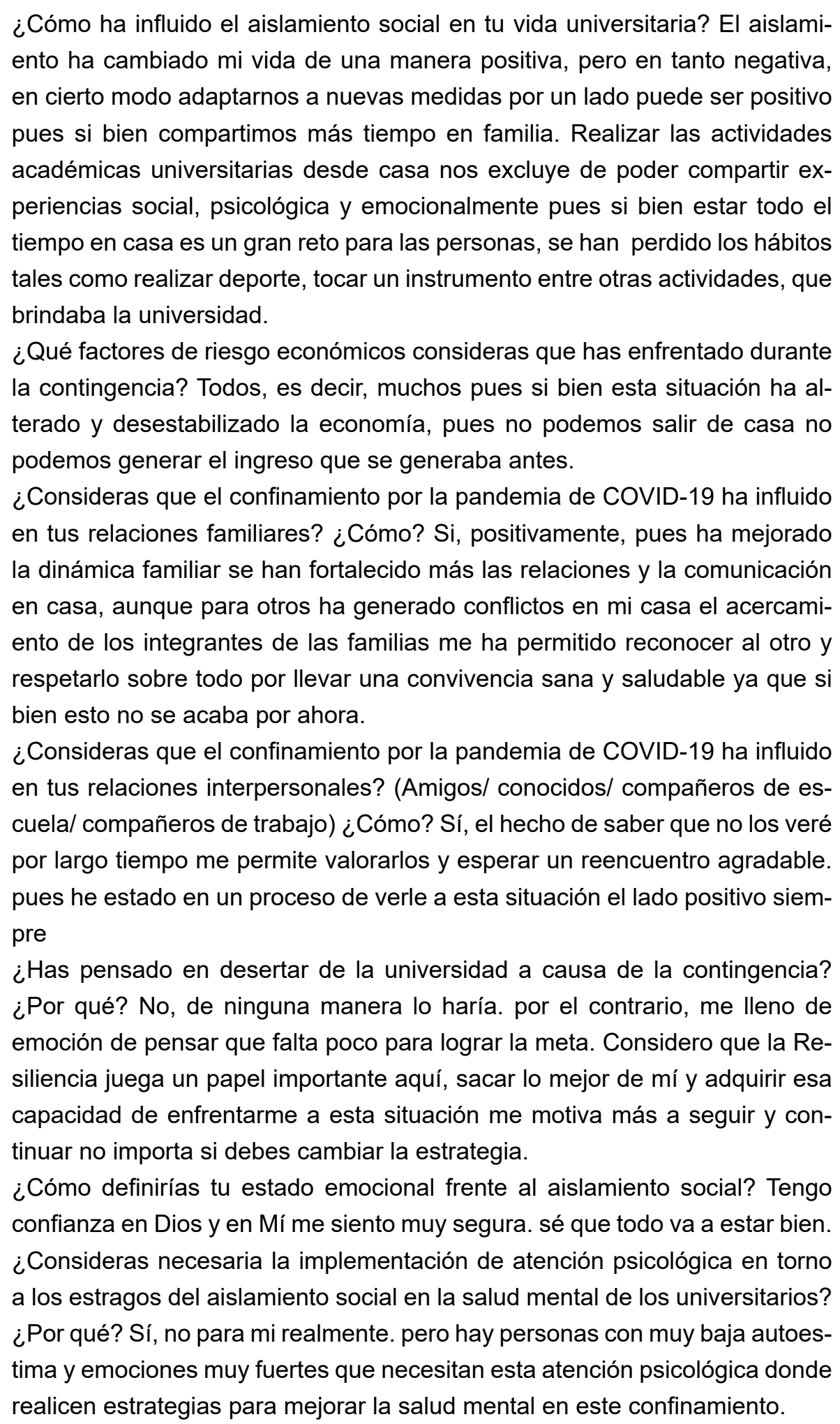 \\
\hline
\end{tabular}


¿Tu universidad ha implementado programas de intervención ante crisis psicológicas respecto al aislamiento social? ¿Cuáles? en la oficina de bienestar universitario, a menudo realiza acompañamiento psicosocial, a través de conversatorios plataforma meeting e Instagram

¿Te encuentras satisfecho respecto a la nueva modalidad de enseñanzaaprendizaje implementada por tu universidad? ¿Por qué? Al principio fue muy difícil en el manejo de la plataforma y han realizado cambios positivos, gracias a una resolución de rectoría los profesores son flexibles para que cada estudiante del alma mater cumplan con sus actividades y puedan culminar su proceso.

Elaboración investigadores Brito Carrillo, Pinto Aragón \& Muñoz Roja, 2021

\section{ANÁLISIS CASO 3}

De manera reiterativa, manifiesta, que presenta estrés y ansiedad, esto a causa de la preocupación por los procesos universitarios, en estos presenten dificultades tecnológicas ya que el internet tiene deficiencias en el área en la que se encuentra y regularmente se quedan sin energía eléctrica. Esto afecta directamente su rendimiento universitario, además de que varias actividades escolares han sido canceladas, lo que genera "vacíos" en su educación.

Menciona que es necesaria la atención psicológica en el ámbito educativo, la atención a las barreras en el aprendizaje, así como capacitaciones por parte de las instituciones educativas para el manejo de las herramientas tecnológicas que se encuentran a su alcance. En cuanto a su experiencia durante el confinamiento encuentra limitación afectivamente, muestra síntomas de estrés, ansiedad e insomnio. De igual manera menciona que le ha ayudado como crecimiento personal, así como mejorar su asertividad familiar, resiliencia y empatía. En el ámbito económico se encuentra estable, ya que ha recibido apoyos municipales. Además de que su universidad les ofreció la matrícula gratis. 


\section{CASO 4}

\begin{tabular}{|c|c|}
\hline Datos & Experiencia a través del aislamiento social por COVID-19 \\
\hline $\begin{array}{l}\text { Género: Femenino } \\
\text { Edad: } 23 \text { Años } \\
\text { Estado civil: Casada } \\
\text { Actividad laboral } \\
\text { del esposo Técnico } \\
\text { Sistema } \\
\text { Programa Trabajo } \\
\text { social }\end{array}$ & $\begin{array}{l}\text { ¿Cómo ha influido el aislamiento social en tu vida universitaria? Bastante duro } \\
\text { ya que uno debe reinventarse ante la situación } \\
\text { ¿Qué factores de riesgo económicos consideras que has enfrentado durante } \\
\text { la contingencia? A nivel económico me he enfrentado a tratar de entender lo } \\
\text { que está pasando a que ya no queda espacio para cosas innecesarias solo } \\
\text { luchar por mantenerse vivo } \\
\text { ¿Consideras que el confinamiento por la pandemia de COVID-19 ha influido } \\
\text { en tus relaciones familiares? ¿Cómo? Si ha influido el confinamiento en mis } \\
\text { relaciones interpersonales ya que me he alejado bastante de ellos por pensar } \\
\text { que cada quien tiene muchos problemas } \\
\text { ¿Cómo te has sentido respecto a tu vida universitaria durante la contingencia? } \\
\text { (Emociones/Sentimientos/Pensamientos) Confundida sin saber que pueda } \\
\text { pasar } \\
\text { ¿Has pensado en desertar de la universidad a causa de la contingencia? } \\
\text { ¿Por qué? Si lo he pensado porque no me gusta casi la modalidad virtual sino } \\
\text { presencial siento que uno adquiere más conocimiento } \\
\text { ¿Cómo definirías tu estado emocional frente al aislamiento social? Confundida } \\
\text { ¿Consideras necesaria la implementación de atención psicológica en torno } \\
\text { a los estragos del aislamiento social en la salud mental de los universitarios? } \\
\text { ¿Por qué? Si porque muchas personas no son tan resilientes ante las } \\
\text { situaciones } \\
\text { ¿Tu universidad ha implementado programas de intervención ante crisis } \\
\text { psicológicas respecto al aislamiento social? ¿Cuáles? No } \\
\text { ¿Te encuentras satisfecho respecto a la nueva modalidad de enseñanza- } \\
\text { aprendizaje implementada por tu universidad? ¿Por qué? No me gusta mejor } \\
\text { presencial pero también sé que es cuestión solo de acostumbrarse }\end{array}$ \\
\hline
\end{tabular}

Elaboración investigadores Brito Carrillo, Pinto Aragón \& Muñoz Roja, 2021

\section{ANÁLISIS CASO 4}

De manera afirmativa expresa, que existe un antes y un después de la pandemia. impactante, afirma que la sociedad no estaba preparada y asimilar la situación es realmente difícil. Académicamente tuvo grandes problemas para continuar con sus estudios ya que no cuenta con computadora, así que tomaba las clases a través de su celular, sin embargo, en este no tenía internet por lo cual debía realizarse recargas telefónicas, esto implicaba un gasto que no podía realizar a causa del desempleo que ocasionó la pandemia, para ello lograr acceder a sus clases algunos docentes le hacían el favor de otorgarle recargas. Cuenta que en su contexto logró notar un desorden emocional, algunos de sus compañeros de la universidad desistieron a causa de las complicaciones que atravesaban. Afirma que la universidad necesita iniciativas de apoyo psicológico y de motivación para que sus estudiantes continúen. 
Económicamente se ha apoyado de familiares y del estado, ya que actualmente ella se encuentra desempleada. Menciona que a nivel cultural ha visto grandes consecuencias negativas, una de ellas es el rompimiento de los modos de despedir a los fallecidos. Las personas no pueden vivir su duelo, las dolencias aumentan y con ellas la ansiedad. Es necesario apoyar a la familia.

Familiarmente su rutina ha cambiado de manera significante, en las condiciones en las que se encuentra busca nuevas técnicas de recreación a hijos para que estos puedan entender y asimilar lo que está ocurriendo a su alrededor, su esposo se quedó sin empleo por lo cual ahora pasa todo el tiempo en casa, esto denotó una sobreprotección a su hijo lo cual genera discusiones en la pareja, por otro lado, ambos sufren de insomnio a raíz del confinamiento. Opina que es necesaria una red de apoyo psicológico por parte de las instituciones educativas, sostiene que los docentes no ven la nueva realidad lo cual dificulta el proceso de enseñanza aprendizaje.

\section{CASO 5}

\begin{tabular}{|l|l|}
\hline Datos & Experiencia a través del aislamiento social por COVID-19 \\
\hline Eénero: Masculino & ¿Cómo ha influido el aislamiento social en tu vida universitaria? Me ha \\
Estado civil: soltero & afectado de manera significativa, el estrés y la ansiedad se presentan todos \\
Convive con su & los días. \\
madre Biológica es & ¿Qué factores de riesgo económicos consideras que has enfrentado durante \\
Inestabilidad & la contingencia? Mis padres perdieron su empleo por lo cual tuvimos que \\
económica y laboral & recortar gastos en casa. \\
& ¿Consideras que el confinamiento por la pandemia de COVID-19 ha influido \\
& en tus relaciones familiares? ¿Cómo? Sí, peleo mucho con mi hermano. \\
& ¿Consideras que el confinamiento por la pandemia de COVID-19 ha influido \\
& en tus relaciones interpersonales? (Amigos/ conocidos/ compañeros de \\
& escuela/ compañeros de trabajo) ¿Cómo? Sí, ya que nos ha distanciado \\
bastante \\
\\
¿Cómo te has sentido respecto a tu vida universitaria durante la contingencia? \\
(Emociones/Sentimientos/Pensamientos) Estresada, muy ansiosa. \\
& ¿Has pensado en desertar de la universidad a causa de la contingencia? ¿Por \\
& $\begin{array}{l}\text { qué? No } \\
\text { ¿Cómo definirías tu estado emocional frente al aislamiento social? Estresante } \\
\text { ¿Consideras necesaria la implementación de atención psicológica en torno } \\
\text { a los estragos del aislamiento social en la salud mental de los universitarios? } \\
\text { ¿Por qué? Sí, ya que eso nos ayudaría a mantener una salud mental estable. } \\
\text { ¿Tu universidad ha implementado programas de intervención ante crisis } \\
\text { psicológicas respecto al aislamiento social? ¿Cuáles? No } \\
\text { ¿Te encuentras satisfecho respecto a la nueva modalidad de enseñanza- } \\
\text { aprendizaje implementada por tu universidad? ¿Por qué? No, ya que la } \\
\text { calidad en la educación ha disminuido }\end{array}$ \\
\hline
\end{tabular}

Elaboración investigadores Brito Carrillo, Pinto Aragón \& Muñoz Roja, 2021 


\begin{tabular}{|l|}
\hline \multicolumn{1}{|c|}{.ANÁLISIS CASO 5 } \\
\hline Menciona que con la llegada del confinamiento su vida ha sido afectaba de manera significativa, ya \\
que en un inició rompió con sus esquemas cotidianos, pasando de estar la mayor parte del tiempo \\
fuera de casa, realizando prácticas ya acudiendo a clases presenciales. Al inicio se mantuvo en la \\
incertidumbre de lo que ocurriría con sus estudios ya que sus docentes no se contactaban con los \\
estudiantes. Cuenta que la mayor parte de ellos solo les perdieron trabajos por lo cual sintió que no \\
logró un buen aprendizaje. En el ámbito económico su familia se vio afectada ya que su madree \\
perdió su empleo por lo cual tuvieron que reducir los gastos mientras buscaban nuevas maneras de \\
obtener ingresos económicos \\
\hline En sus relaciones interpersonales sufrió un gran distanciamiento con sus amigos y compañeros \\
de la escuela, además de que dejó de ver a su familia paterna, a la cual veía cada vacación, pero \\
a causa del confinamiento no logró dejar la ciudad para poder visitarlos. Anímicamente manifestó \\
problemas de ansiedad, estrés, preocupación e intolerancia, esto lo vio manifestado en insomnio y \\
mal humor. Los problemas en casa aumentaron ya que peleaba regularmente con su hermano, todo \\
esto la llevó a padecer de depresión. Afirma que es necesario un acompañamiento psicológico por \\
parte de las instituciones académicas para sus estudiantes, esto con el fin de garantizar un apoyo \\
multidisciplinar que les permita mantener una salud mental estable y continuar con los estudios de \\
una mejor manera.
\end{tabular}

\section{CONCLUSIONES}

A partir de la información obtenida a través de las entrevistas semiestructuradas, así como de los conversatorios y entrevistas, se obtuvo que la población estudiantil perteneciente al nivel superior ha presentado diversos estragos en su salud mental, entre los cuales se destacan los siguientes: ansiedad, estrés preocupación enojo, miedo, insomnio, irritación, tristeza

Esto se debe a múltiples factores de riesgo en diversas áreas. Dentro de la económica se encuentran la pérdida de empleo por dos razones: la primera es el cierre de empresas debido al confinamiento y la segunda la reducción de aforo que implicó el recorte de personal; por otro lado, la reducción de salarios a los empleados. En el ámbito familiar es importante destacar el incremento de problemáticas a causa de la adaptación a la nueva realidad, la cual implica compartir los espacios por más tiempo, el rompimiento de esquemas de la cotidianidad generó estrés y ansiedad en las personas, lo cual afectó directamente sus relaciones intrafamiliares. Con respecto a las relaciones interpersonales, estas se vieron impactadas a causa del confinamiento, ocasionando así un distanciamiento de ellas.

\section{REFERENCIAS BIBLIOGRÁFICAS}

Arias, F. (2016). El Proyecto de investigación. Introducción a la Metodología Científica. $7 \mathrm{ma}$ ed. Venezuela: Editorial Episteme.

Arias F (2012). El Proyecto de Investigación Introducción a la metodología científica.

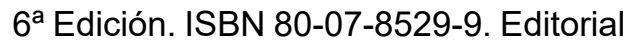
Episteme, C.A. Caracas Venezuela

Ballesteros, B. (2014). Taller de investigación cualitativa. Madrid, España: Universidad nacional de educación a distancia UNED

Bernal C A., (2010) Metodología de la investigación. Tercera edición. PEARSON EDUCACIÓN. ISBN: 978- 
958-699-128-5. Área: Metodología. Colombia.

Berenguera $A$, Fernández $M$, Pons $M$, Pujol E, Rodríguez D y Saura S. (2014) Escuchar observar y comprender. Recuperando la narrativa en las Ciencias de la Salud. Aportaciones de la investigación cualitativa. Barcelona: Institut Universitari d'Investigació Primària Jordi Gol.

Cao, W., Fang, Z., Hou, G., Han, M., Xu, X., Dong, J. \& Zheng, J. (2020). The psychological impact of the COVID-19 epidemic on college students in China. julio 18, 2020, de Psychiatry Research Sitio web: https://doi.org/10.1016/j. psychres.2020.112934

Chárriez, M. (2012). Historias de vida: Una metodología de investigación cualitativa. Griot (p.51)

Cotacachi, D. y Grigera, A. (2020). Pandemia 2020: Respuestas urgentes y diferenciadas para pueblos indígenas, julio 26, 2020, de Banco Interamericano de Desarrollo Sitio web: https:// blogs.iadb.org/igualdad/es/covid-19respuestas-pueblos-indigenas/

Cullari, S. (2001). Fundamentos de Psicología Clínica. México: Pearson.

El Heraldo. (2020). El impacto del coronavirus en las universidades. EL HERALDO, 12.

El impacto del Coronavirus en las universidades. El lesalc-Unesco advierte que la sustitución de las clases presenciales por la modalidad virtual está generando dificultades porque los contenidos no. https://www. elheraldo.co/informes-comerciales/ el-impacto-del-coronavirus-en-lasuniversidades-730425
Ellis, R. E. R., Seal, M. L., Simmons, J. G., Whittle, S., Schwartz, O. S., Byrne, M. L., \& Allen, N. B. (2017). Longitudinal Trajectories of Depression Symptoms in Adolescence: Psychosocial Risk Factors and Outcomes. Child Psychiatry and Human Development, 48(4), 554571. https://doi. org/10.1007/s10578016-0682-z

Galimberti, U. (2002). Diccionario de Psicología. Buenos Aires, Argentina: Siglo XXI Editores.

Fuster, D. E. (2019). Investigación cualitativa: Método fenomenológico hermenéutico. Scielo, p. 221 - 229. doi:http://dx.doi. org/10.20511/pyr2019.v7n1.267

Hernández S. R. Metodología de la Investigación. Quinta edición derechos reservados (C) 2010, 2006, 2003, 1998, 1991 respecto a la quinta edición por: McGraw-Hill / Interamericana Editores, S.A. DE C.V. ISBN: 978-607-15-0291-9. México

Hernández, R., Fernández, C., Baptista, P. (2014). Metodología de la investigación. México: Mc Graw Hill

Huarcaya-Victoria J. Consideraciones sobre la salud mental en la pandemia de COVID-19. Rev Peru Med Exp Salud Publica. 2020; 37(2): 32734. doi: https://doi.org/10.17843/ rpmesp.2020.372.5419

Li, J., $\square$ eng, Y. L., \& Foo, S. (2015). Depression and Psychosocial Risk Factors among Community-Dwelling Older Adults in Singapore. Journal of Cross-Cultural Gerontology, 30(4), 409-422. https:// doi.org/10.1007/ s10823-015-9272-y 
López, E. (2012). Programas de implicación paterna en Educación Infantil. Revista Complutense de Educación, 6(1), 115130.

Martínez, L. C., \& Paladinez, M. Y. (2016). Trastornos Afectivos y su Incidencia en el Rendimiento Académico de los Estudiantes de la Escuela de Educación Básica "Paul Percy Harry Bryan" de la Ciudad de Machala Perteneciente a la Ciudadela El Bosque (Tesis de grado) no publicado. Obtenido de http://repositorio.utmachala.edu.ec/ bitstream/48000/4599/1/CD000152016-TESIS\%20COMPLETA.pdf

Martínez-Garcés, J y Garcés-Fuenmayor, J. (2020). Competencias digitales docentes y el reto de la educación virtual derivado de la covid-19. Educación y Humanismo, 22(39), 1-16. https://doi. org/10.17081/eduhum.22.39.41141

Malave, I. C. (19 de 06 de 2020). vocespr. Obtenido de https://www.vocespr.org/ post/el-estres-en-tiempo-de-pandemia

Monje, C. (2011). Metodología de la investigación cuantitativa y cualitativa, guía didáctica. Neiva, Colombia: Universidad Surcolombiana.

Monge, E (2010) El Estudio de Casos como Metodología de Investigación y su importancia

en la dirección y Administración de Empresas. Revista Nacional de administración,

1 (2): 31-54 Julio-Diciembre.

Morales, F. (2010). Estudio de salud mental en estudiantes universitarios de la Facultad de Ciencias de la Educación de la Universidad De Carabobo. (Tesis doctoral). Universidad Autónoma de
Madrid, Madrid.

Organización Mundial de la Salud. (2004). ¿Qué es la salud mental? En Invertir en Salud Mental (p.7). Suiza: Organización Mundial de la Salud.

Organización Mundial de la Salud. (2020 Nuevo coronavirus. Disponible en https://www. minsalud.gov.co/Paginas/default.aspx

Organización Mundial de La Salud. (OMS, 2020). En tiempos de estrés, haz lo que importa: una guía ilustrada [Doing what matters in times of stress: an. Obtenido de https://apps.who.int/iris/bitstream/ha ndle/10665/336218/9789240009561spa.pdf

Peiró R (2021, 05 de enero). Psicología de la salud. Economipedia.com https:// economipedia.com/definiciones/ psicologia-de-la-salud.html

Piña, J y Rivera, B (2006). Psicología de la salud: algunas reflexiones críticas sobre su Qué y su Para Qué. Universitas Psychologica, 5(3),669679. ISSN: 1657-9267. Disponible en: https://www.redalyc.org/articulo. oa?id=647/64750319

Shereen, M. A., Khan, S., Kazmi, A., Bashir, N., \& Siddique, R. (2020). COVID-19 infection: Origin, transmission, and characteristics of human coronaviruses. Journal of Advanced Research, 24, 91-98. https:// doi.org/10.1016/j.jare.2020.03.005

Silveira, D., Colomé, C., Heck, T., Nunes, M. y Viero, V. (2015). Grupo focal y análisis de contenido en investigación cualitativa. Index de Enfermería, 24(12), 71-75. https://dx.doi.org/10.4321/ $\underline{\mathrm{S} 1132-12962015000100016}$ 
Starks Hy Brown ST (2007) Choose your method:

a comparison of phenomenology, discourse analysis, and grounded theory. Qualitative health research 17 (10): PP. 1372-1380

Organización de las Naciones Unidas para la Educación, la Ciencia y la Cultura (UNESCO, 2020). Las universidades abordan el impacto de COVID-19 en los estudiantes desfavorecidos. julio 29 , 2020, de UNESCO Sitio web: https:// es.unesco.org/news/universidadesabordan-impacto-covid-19-estudiantesdesfavorecidos

Ramos, C. (2017). Los Paradigmas de la Investigación Científica. Avances en Psicología, 23(1), 9-17.

Real Academia Española. (2016). Real Academia Española. Recuperado el 5 de febrero de 2016, de http://www.rae. es/.

Rull, A. (4 de 07 de 2019). Periodico.com. Obtenido de https://www.elperiodico. com/es/ser-feliz/20190313/eliminarexceso-preocupaciones-7338764

Valero, N., Vélez, M., Durán, A., \& Portillo, M. (2020). Afrontamiento del COVID-19: estrés, miedo, ansiedad y depresión? Enferm Inv, 5(3), 63-70. Obtenido de https://core.ac.uk/download/ pdf/337425623.pdf

Wu, Z., y McGoogan, J. M. (2020). Characteristics of and Important Lessons from the Coronavirus Disease 2019 (COVID-19) Outbreak in China: Summary of a Report of 72314 Cases from the Chinese Center for Disease Control and Prevention. In JAMA - Journal of the American Medical Association, 323(13), 1239-1242). https://doi.org/10.1001/jama.2020.2648 